\title{
GESNERUS
}

Vierteljahrsschrift für Geschichte der Medizin und der Naturwissenschaften Revue trimestrielle d'histoire de la médecine Jahrgang/Vol. $25 \quad 1968$ Heft/Fasc. 3/4

\section{Neue Funde zum Thema: L'homme machine und Albrecht Haller}

Von Erich Hintzsche, Bern

\section{Einleitung}

Bücher und Broschüren wissenschaftlichen Inhaltes, die anonym oder mit fingierten Autorennamen veröffentlicht wurden, waren um die Mitte des 18. Jahrhunderts keine Seltenheit. Sie beunruhigten vor allem die Rezensenten, die für die gerade damals vermehrt erscheinenden literarischen Zeitschriften arbeiteten. Überprüft man verschiedene Besprechungen und Nachrichten, die das gleiche Werk betreffen, so finden sich neben unrichtigen Vermutungen auch genau belegte und deshalb zuverlässig erscheinende Mitteilungen über die Person des anonymen Autors. Besonders auffällig ist aber der Fall, wo eine solche Angabe zunächst falsch war, dann berichtigt wurde und schließlich doch wieder die ursprüngliche falsche Form als gültig anerkannt ist. Als solch ein Beispiel mag das Werk L'homme plus que machine dienen, das 1748 angeblich in London publiziert wurde. In den Göttingischen Zeitungen von gelehrten Sachen erschien in der 3.Zugabe zum Mai 1748 (61. Stück, S.486-488) eine erste Besprechung, die nach GuthкE (1962, S.138) von Haller stammt. Darin heißt es: «Das eigneste bey der ganzen Sache ist, daß der Hr. de La Mettrie selber der Verfasser dieser Schrift, nach zuverlässigen Nachrichten ist, und durch dieselbe den Vorwurf [hat $]^{1}$ ablehnen wollen, den er sich bey allen Gott- und Wahrheitliebenden durch die starke Vermuhtung zugezogen, daß er der Verfasser des homme Machine seye.» Schon wenige Wochen später, am 24. Juni 1748,

1 Eckige Klammern in Zitaten bezeichnen Zusätze oder sprachliche Abänderungen des Verfassers. 
folgte dann aber eine Korrektur (gleiche Zeitschrift, 70.Stück, S. 560): «Ein Holländischer junger Gelehrter, dessen Anfangsbuchstaben wir blos mit E.L. anzeigen wollen, hat an uns geschrieben, und uns versichert, daß er der Verfasser des homme plus que machine wäre, von dem andere Gönner uns benachrichtigt [hatten], daß sie des Hrn. La Mettrie eigene Arbeit [im Original Plural] wäre. Ob uns nun wohl des Hrn E.L. ganz genaue Bekanntschaft und Verknüpfung mit dem Verfasser des homme machine wohl bekannt ist, so haben wir doch um unsre Unpartheylichkeit ihm zu bezeugen, seine gethane Versicherung bekannt zu machen nicht unterlassen wollen.» ${ }^{2}$ Es liegt nahe anzunehmen, daß Haller selbst diese berichtigende Mitteilung in die Göttingischen Zeitungen von gelehrten Sachen hat aufnehmen lassen, deren Direktor er damals war. Von seiner genauen Kenntnis des Sachverhaltes zeugt jedenfalls eine Besprechung des 1752 in La Haye erschienenen Eloge de La Mettrie (Göttingische Zeitungen von gelehrten Sachen auf das Jahr 1753, 32. Stück, S. 303-304, datiert 12. März 1753, im Berner Exemplar mit einem handschriftlichen $\mathrm{H}$ am Rande versehen, also sicher aus Hallers Feder stammend). Am Schluß dieser Rezension sind den im Werke angeführten Publikationen einige Notizen beigefügt. Darin heißt es: «Der homme plus que Machine ist eine Arbeit des Leidenschen Buchhändlers Elie Luzac's und nicht des Hrn. v. Haller.» Hier steht also der volle Name des wahren Autors. Trotzdem schrieb Haller in seiner Bibliotheca anatomica (Vol. II, p. 370, 1777) im Kapitel Julianus Offray vulgo de La Mettrie den homme plus que machine wieder diesem zu, er kehrte also zu seiner ursprünglichen, jedoch falschen Ansicht zurück. Möglicherweise hat dazu beigetragen, daß in einer Ausgabe der CEuvres philosophiques von De LA Mettrie (Amsterdam 1760) das Werk L'homme plus que machine enthalten ist (Guthke, 1962, S.138); Guthke mißt diesem Umstand aber wenig Bedeutung für die Urheberschaft zu, da die erwähnte Edition neun Jahre nach dem Tode des Verfassers erschien. Eine andere Ausgabe der Euvres philosophiques (Amsterdam 1753), die ich einsehen konnte, enthält die oben zitierte Schrift nicht; Haller besaß die 1.Auflage (Amsterdam $1751)$ in der nach Zimmermanns Zitat $(1755$, S. 233, Fußnote $\langle\mathrm{e}\rangle)$ die Studie L'homme plus que machine gleichfalls fehlt.

2 Diese nach Göttingen gelangte Mitteilung stammte von Elie LuzAC, dem Verleger des Homme machine; sein Brief ist im handschriftlichen Verzeichnis der Haller-Korrespondenz angeführt, aber wie manche andere Schreiben früher entwendet worden, er befindet sich heute in der Handschriften-Abteilung der Bibliothek der Reichs-Universität Leiden; Geschichte und Wortlaut bei GuthKe, 1962, S.141-143. 
Obwohl das angeführte Beispiel mein Thema nur am Rande berührt, schien es mir doch als Beleg dafür geeignet, daß der gleiche Rezensent für dasselbe anonym erschienene Werk - zeitweise schwankend - verschiedene Autorennamen anführt. Daraus wird ersichtlich, daß gegen derartige Angaben in den literarischen Gazetten des 18. Jahrhunderts eine gewisse Skepsis am Platze ist. Man sollte nach Möglichkeit versuchen, solche Notizen noch auf andere Weise zu sichern. In dieser Überzeugung habe ich das Thema «L'homme machine und Albrecht Haller» wieder aufgenommen. Neue Quellen haben mir geholfen, falsche Angaben zu berichtigen und manche Unsicherheiten abzuklären. Entsprechend der Herkunft des neuen Materiales ist die Studie in zwei Teile gegliedert, die sich gegenseitig ergänzen. Eine Schlußbetrachtung wird die Einzelergebnisse zusammenfassen und miteinander verknüpfen.

\section{L'homme machine in Briefen von P.G. Werlhof an A. Haller}

Da sich früher in Hallers Korrespondenz erste Hinweise auf einen Mitarbeiter von de La Mettrie fanden, auf den gewisse Angaben im Werk L'homme machine zurückgehen (Hintzsche, 1966), lag es nahe, auch andere Teile von Hallers Briefwechsel mit seinen engeren Freunden auf diese Frage zu prüfen. Auffälligerweise enthalten die an seinen Jugendfreund Johannes Geßner gerichteten Mitteilungen nichts Derartiges. Andere Briefreihen ihm Nahestehender, z.B. von Tissot und Bonnet, beginnen erst in den Jahren nach Hallers Rückkehr in die Heimat. Damals hatte der am 11. November 1751 erfolgte Tod de La Mettries schon weitere Diskussionen überflüssig gemacht. Schließlich wies mich Herr Dr. H. HäBerLI von der Burgerbibliothek Bern noch auf die Briefe Werlhofs hin, die - weil separat gebunden - leicht übersehen werden; seine Anregung, für die ich sehr zu danken habe, erwies sich als recht fruchtbar.

Über Haller (1708-1777) sind biographische Angaben überflüssig, da sie leicht zugänglich sind. Paul Gottraried Werlhof (1699-1767), der Schreiber der hier benutzten Briefe, gilt als ein bedeutender Förderer der Universität Göttingen. Von seiner Freundschaft mit Haller zeugen 1585 an diesen gerichtete schriftliche Mitteilungen aus den Jahren 1736-1767. Nach Pagels Bericht im Biographischen Lexikon hervorragender Ärzte stammte Werlhof aus Helmstädt, wo er am 24. März 1699 geboren wurde; am gleichen Ort studierte er bei Meibom und Heister Medizin. Schon 1721 
begann er in Peine bei Hildesheim als Arzt zu praktizieren. Den Doktortitel erwarb er 1723 in Helmstädt. 1725 verlegte er seine Praxis nach Hannover, wo ihm sein wissenschaftlicher und ärztlicher Eifer bald einen geachteten Namen und großen Anhang verschafften. Nachdem er einen Ruf an die Universität Helmstädt abgelehnt hatte, wurde er $1740 \mathrm{Kgl}$. Leibarzt. Als geübter Schriftsteller und Verfasser von Gedichten war er in der Lage, Haller vielfach in sprachlichen Fragen zu beraten. Er unterstützte beim hannoverschen Ministerium auch Hallers Anliegen, die die Anatomie, den Botanischen Garten und sonstige Göttingische Universitätsprobleme betrafen. Von dem bis zu Werlhofs Tod am 26. Juli 1767 fortgeführten Briefwechsel mit Haller werden hier nur seine Mitteilungen aus den Jahren 1747-1752 berücksichtigt. Gegenbriefe Hallers fehlen, da Werlhof dessen Schreiben verbrannte, wie den nachfolgenden Briefauszügen zu entnehmen ist.

Die eben genannte zeitliche Begrenzung wird am besten verständlich durch ein paar Hinweise auf de La Mettries Leben und Werk; sie beschränken sich auf Angaben, die zum Thema Beziehung haben. Eine ausführliche Biographie, die mir auch in psychologischer Hinsicht gut fundiert scheint, findet sich bei Vartanian (1960, S.1-12). Geboren am 25. Dezember 1709 in Saint-Malo war Julien Offray de La Mettrie nur um ein Jahr jünger als Haller. Er erhielt seine Grundschulung in Coutances und Caën, neigte in seinen Jugendjahren theologischen Studien zu, wurde dann aber doch Arzt. Er studierte Medizin in Paris und Reims, an diesem Ort doktorierte er auch. 1733/34 setzte er seine Ausbildung bei Boerhaave in Leiden fort, der ihn sehr geschätzt haben soll (Biogr. méd., Paris 1824, T.6, p.266). Nach Hause zurückgekehrt, widmete er sich neben der ärztlichen Praxis durch Übersetzungen der Verbreitung der BoERHAaveschen Schriften, vor allem der Institutions de Médecine, die er mit einem Kommentar versah (8 vol., Paris 1743-1750). Da Haller selbst Boerhaaves Praelectiones academicae in proprias institutiones rei medicae (7 vol., Gottingae 1739-1744) herausgegeben hatte, erkannte er bei der Besprechung der ersten drei Bände der Übersetzung von de La Mettrie (Göttingische Zeitungen von gelehrten Sachen 1745, 46. Stück, S. 377-378), daß dieser zwar zugab, Hallers Kommentare benutzt zu haben, die darin mitgeteilten Beobachtungen aber oft als seine eigenen anführte und nur vereinzelt Hallers Namen dabei nannte. Haller wies auch mancherlei Fehler in de La Mettries Buch nach und schloß, dieser hätte sich eines fremden Werkes bedient und dabei weder die Aufrichtigkeit noch die Einsicht gebraucht, die man von ihm verlangen könne. 
Eine andere Besprechung Hallers (Göttingische Zeitungen von gelehrten Sachen 1747, 51.Stück, S.413-415) betraf de La Mettries Histoire naturelle de l'Ame, La Haye 1745; das Büchlein sollte angeblich von einem Engländer Dr. Charp verfaßt und von einem ungenannten französischen Mitglied der Akademie übersetzt worden sein. Haller erkannte, daß es in Frankreich entstanden sein mußte und hielt de La Mettrie für den Autor. Er wies ihm nach, daß vieles aus BoerhaAves Institutiones und Hallers dazu publizierten Erläuterungen übernommen sei, und sprach im Zusammenhang damit geradezu von «Diebstahl». Die Reaktion de La Mettries ließ nicht auf sich warten, sie erschien in der Form des anonymen Werkes L'homme machine (Leyde 1748). Das Büchlein wurde so berühmt, daß es Garrison und Morton in die Medical Bibliography aufnahmen (1. ed.1943, Nr. 685; 2. ed.1961, Nr. 586). Diese Arbeit war in Leiden entstanden, da de La Mettrie infolge von Streitigkeiten mit der Pariser Medizinischen Fakultät vorgezogen hatte, 1746 Frankreich zu verlassen und nach Holland zu übersiedeln.

In den Göttingischen Zeitungen von gelehrten Sachen stehen zwei Rezensionen der Broschüre L'homme machine; die erste noch im Dezember 1747 - erschienene findet sich im 108. Stück auf S. 905-907, eine zweite ausführlichere Besprechung folgte in der 1. und 2. Zugabe zum Mai 1748 (52. und 54.Stück, S.409-412 und 425-428). Auf einen Ruf König Friedrichs II. von Preußen hin hatte sich de La Mettrie mittlerweile nach Berlin begeben, wo er am 7. Februar 1748 eintraf. Er wurde dort Mitglied der Akademie, publizierte sehr fleißig, starb aber - wie erwähnt - schon am 11. November 1751. Unsere Frage lautet: Finden sich in Werlhofs Briefen Hinweise auf die Person des oder der Rezensenten der Schrift L'homme machine, und sind ihnen sonstige Hinweise zu entnehmen, die im Zusammenhang mit dieser Publikation stehen?

Werlhof an Haller $(59 / 185)^{3}$ : $\quad$ Hanover, Dec. $8^{\text {th }}(17) 47$

There is a small Book, entitled l'homme machine, dedicated to You by the Author, who stiles himself Your Friend and Disciple, a poor Materialist, Pyrrhonian and inclined rather to Atheism, than Deism, by Moralogisms drawn from physical Observations, all ill ordered, with some Sparrs of Wit. He is, it seems, a Physician and a Switzer, saying somewhere: nous avons eu en Suisse un Baillif, nommé Mr. Steiguer de Witighofen, of whom he says, that after dinner, he was able to hang any innocent Man, though otherwise he was a just

${ }^{3}$ Die den Briefen Werlhofs an Haller beigefügten Zahlen bedeuten Band- und Briefnummer in der Haller-Korrespondenz. 
and indulgent Judge. Mr. Augspurg ${ }^{4}$, who, at Mecenas's Order $^{5}$ has made an abstract of the Book, shew'd it me last Night. The Title bears à Leide de l'imprimerie d'Elie Luzac fils 1748. But it is come hither from Berlin ${ }^{6}$. Mr. Augspurg had already sent his Abstract to Mecenas, so I have not seen the same, nor perused the Book. Only looking into it for a Quarter of an Hour, I found it, as I said, and the Author an impudent fellow...

4 Adelung (1784, Spalte 1244) nennt Johann Christian Augsburg als einen Rechtsgelehrten, der um die Mitte des 18. Jahrhunderts lebte; seine Dissertation erschien 1740 in Marburg.

Nach Auskunft des Niedersächsischen Staatsarchives Hannover erhielt Johann Christoph Augspurg am 26. April 1742 die Stelle eines Secretarius extraordinarius bei der Justizkanzlei in Hannover. Gerade während der Zeit, in der wir uns mit ihm zu beschäftigen haben, wurde er - «wegen seiner besonderen Wissenschaft, Fleißes und übrigen Geschicklichkeit bekannt und angerühmt» - im Januar 1748 zu einem der Kammersekretäre vorgeschlagen und ernannt. Er rückte schließlich in die Erste Sekretärsstelle auf und blieb bis $1770 \mathrm{im} \mathrm{Amt;} \mathrm{gestorben} \mathrm{ist} \mathrm{er} \mathrm{im} \mathrm{September} 1771$.

Über Augspurgs Fähigkeiten und seine Tätigkeit als Rezensent finden sich Angaben in zwei weiteren Briefen, die sich jedoch auf ein anderes Werk beziehen:

Werlhof an Haller (60/23): $\quad$ Hannover March $11^{\text {th }}$ [17] 48

Good Mr. Augspurg, one of the most honest Men I know, was ordered I think by Mecenas (siehe Fußnote 5) to make a honourable Abstract of Simonetti, and knew not to keep Measure. His learning is civil- and public Law, Mathematicks, and Wolffian Philosophy, and he writes well in Latin and german. French reading, modern litterary History is not his Business. He thinks, I believe, he dares not refuse Mecenas, who sends him Novelties, to make Abstracts of, be they in or out the Way of his Abilities, though he thinks perhaps otherwise, and the Art of making Abstracts may be somewhat out. He gets easily into Praises and Disgressions, I see. But I won't lessen Mecenas' Opinion of him, nor deprive him of the Gifts, he receives.

Werlhof an Haller (60/25): $\quad$ Hannover March $18^{\text {th }}[17] 48$

As for Simonetti, I cannot tell if Mecenas had ordered Mr. Augspurg to praise the Man: only I suppose it, because Augspurg makes Abstracts by Orders. I once heard Mecenas say, that he was pleased to read Simonettis writings when the Character of a Divine was published. I see neither Mecenas, nor Mr.Augspurg, when they are well, unless some Friend desires my good Office with the formers, and makes me pay a Visit.

5 Mecenas: Deckname für Adolf Gerlach Freiherr von Münchhausen (1688-1770), der als Kurator die Universität Göttingen leitete; er gründete und förderte die Bibliothek, die wissenschaftlichen Institute und die Göttingischen Zeitungen von gelehrten Sachen. GuthKe (1962 a, S. 30) nennt ihn geradezu deren « eigentlichen Vater»; im gleichen Werk (S.159,Anm.15) weist er darauf hin, daß in Münchhausens Briefen an Haller «ständig von beigelegten Rezensionen von fremder Hand die Rede ist, auf deren raschen Abdruck gedrungen wird». Nur ausnahmsweise ist angegeben, auf welche Bücher sich diese Besprechungen beziehen.

${ }^{6}$ Man vergleiche dazu jedoch den Brief vom 22. Dezember 1747. 
... When I see Mecenas I will endeavour to bring in a Conversation about l'homme machine, though I dare not tell him Augspurg shew'd me the Book. And if Mecenas has it still, no doubt you shall see it. Perhaps I may see him this Morning though Postday ... One Word more on the Materialist. I do not think him born in France by his Stile. He has read Your Poems, whence he borrows several Expressions and pities Voltaire to be unable to read 'em. He quotes La Mettrie. The King of Prussia is a Hero of his. In the Preface the Bookseller says, the Work was sent him from Berlin, with a Petition to send six Copies to Marquiss d'Argens ${ }^{7}$, but that he the Bookseller thinks this very Petition a Mask. However the Book came hither from Berlin ${ }^{6}$. The Author is a Physician, it seems.

Werlhof an Haller (59/188):

Hanover, Dec. $18^{\text {th }}[17] 47$.

I saw Mecenas Friday Morning, and he promised me not only to write to you that Moment as graciously as he spoke to me on Your Account ${ }^{8}$, but also to send You the homme machine. Your Suspicion on Koenig ${ }^{9}$ has a very high Degree of Probability in my Eyes. Be the Author, who he will, he is a bad Man and no Logician at all, only a bold offender to God, against whom he rails, making a Ridicule of the Operation of the Holy Ghost, and excusing Atheism, even concluding by a Sentence without Grounds, there is but one Substance, though formerly he had only set up for a Pyrrhonian between Atheism and Deism.

Werlhof an Haller (59/190):

Hanover Dec. $22^{\text {th }}[17] 47$.

Mecenas has told me in my last Visit, that the Book of the Materialist is not come from Berlin as Mr. Augspurg had made me believe, but from Holland. I am afraid Your Suspicion on König may prove true.

Werlhof an Haller (59/192):

Hanover, Dec. $29^{\text {th }}[17] 47$.

I wish You may not stay longer with the Recension of the Materialist's Pamphlet, least the Leipzig or Hamburg Gazet may come before Yours. Methinks it is a great Commendation to a litterary Gazet, to be beforehand in Notifications and Recensions, even in the first, when the later cannot be done so soon, and may follow afterwards. Your disowning Such an Author, who claims Your Friendship and Instructions, and is read doubtless already by many thousands, cannot come too soon.

Werlhof an Haller (60/1):

Hanover Jan. $5^{\text {th }}[17] 48$

Methinks Mr. Augspurger's Recension of the homme machine is sufficient for the Purpose.

7 Jean Baptiste de Boyer, Marquis d'Argens (1704-1771) war damals Direktor der Berliner Akademie.

${ }^{8}$ Betrifft Geldangelegenheiten.

9 Samuel König (1712-1757) lebte - politischer Gründe wegen aus Bern verbannt damals in Franeker und hielt dort als Professor der Philosophie auch mathematische Vorlesungen. Welche Gründe Haller hatte, ihn als Verfasser des Homme machine zu verdächtigen, ist nicht bekannt. 
I do not believe La Mettrie the sole Author of the homme machine. Some Switzer or German had a Hand in it.

Werlhof an Haller (60/50):

Hanover May $31^{\text {th }}[17] 48$

Good Augspurg! La Mettrie was to cunning for him, and he out of his Sphere. But indeed You need by no Means print what Mecenas send's You of other People's Performances, if You do not find the Things, as they ought to be. Mecenas means well, and Augspurg, who believes he dares not refuse any Task the Minister lays on him and who perhaps thinks himself better versed in French Language, Way of thinking, and litterature than he is, does as good as he can.

Werlhof an Haller (60/56):

Hanover June $17^{\text {th }},[17] 48$

I am curious to see that La Mettries new Performance ${ }^{10}$. The Devil is in the Man, and he believes none.

Werlhof an Haller (60/58):

Hanover June $28^{\text {th }}[17] 48$

Kind Thanks for Clarissa, I did not yet look into, and impudent La Mettrie's Performance, I read a good deel of, a petit maitre in Physick, witty by Chance, because saying all, what falls in his Way, superficial in every part of learning, ill natured, but well acquainted with the Faculty of Paris. The Author of the faculté vangée looks through every Page. He no doubt is acquainted with Your cordate Refusal of his Incense in the homme machine (: a Lesson for Your no betters:) and therefore is grown angry with You. Had You spared his Irreligiousness to pay him for the Compliments he made You in the Preface, he would have spared You, where he spares nobody but Senac and Quesnay.

Werlhof an Haller (60/61):

Hanover July $8^{\text {th }}[17] 48$

I send back today Clarissa and impudent La Mettrie.

Werlhof an Haller (60/93):

Hanover Nov. $4^{\text {th }}[17] 48$

I have not seen the antihomme-machine ${ }^{11}$, nor the Francfort-Gazet, and shall be glad to see the forward Edition of Your Poems etc.

Werlhof an Haller (60/126):

Hanover Febr. 2 [17]50

I have burned all Your Letters, all. They were only for me, and I hope me going to Rest is not so very far off, and who knows how sudden it may be? Sometimes ago I beg'd You to destroy my Letters too. Pray do ... Midstwhile till we live let us go on writing with Trust, but soon burn the Letters.

10 Vermutlich de LA Mettries Schrift L'ouvrage de Pénélope: ou Machiavel en médecine, deren 1.Teil 1748 in Berlin erschien.

11 Welche der gegen den Homme machine gerichteten Schriften gemeint sein kann, bleibt unsicher. 
La Mettrie's Generation of Animals by Chance is a necessary Consequence of his and Spinoza's denying of final Causes. Even the learned Epicurians tought no other Chance, than the eternal necessary Motion of the Atoms, gathering them without any View.

Werlhof an Haller (60/167):

Hanover Aug. 19 ${ }^{\text {th }}[17] 50$

I saw the œuvres philosophiques ${ }^{12}$ of La Mettrie, his foolish Things printed together with a preliminary Discourse to shew the Usefulness of free-thinking, where in a note he rails on Your Protestations against his Dedicacy of the homme machine, and calls it Pedantry. He makes a fine Picture of his King, whom he calls le second Julien.

Werlhof an Haller (60/178):

Hanover Oct. $23^{\text {th }}[17] 50$

La Mettrie is still in Favour with le Second Julien as he stiles the King en guise d'éloge, as You see in the œuvres philosophiques. Voltaire bears openly the Standart of Irreligion.

Werlhof an Haller (60/179):

Hanover Oct. $26^{\text {th }} 1750$.

You may write to me any Thing, without Fear of an ill Use to be made of, as I burn Your Letters, and hope You do so mine.

Werlhof an Haller (61/12):

Hanover Febr. $14^{\text {th }}[17] 52$

The King of Prussia is the Author of the Eloge de La Mettrie read in the Academy.

Bei der großen Zahl der Briefe Werlhofs an Haller erscheinen diese Angaben über das Werk L'homme machine und seinen mutmaßlichen Autor relativ gering. Dabei ist allerdings zu beachten, daß die vorliegende Briefreihe nicht vollständig ist. Eine Zusammenstellung der Zahl der Schreiben Werlhofs für die Jahre 1747-1752 zeigt auffällige Lücken:

$\begin{array}{lrrrrrr}\text { Jahr: } & 1747 & 1748 & 1749 & 1750 & 1751 & 1752 \\ \text { Zahl der Briefe: } & 105 & 101 & 5 & 82 & 0 & 105\end{array}$

Diesen Zustand läßt schon der alte handgeschriebene Katalog der HallerKorrespondenz erkennen; äußerst wahrscheinlich ist deshalb, daß Haller selbst gewisse Briefe vernichtete. 1749 könnten es zum Teil Schreiben gewesen sein, die sich auf Hallers Nobilitierung bezogen, doch deckt diese Vermutung nicht den auf das ganze Jahr verteilten Verlust. Welche Ursache das völlige Fehlen von Briefen aus dem Jahr 1751 bewirkte, bleibt unklar. Chronologische Gründe sprechen dafür, daß Haller vielleicht doch durch Werlhofs wiederholte Bitten veranlaßt wurde, zeitweise dessen Briefe zu vernichten.

12 Die 1.Auflage trägt das Erscheinungsjahr 1751, sie muß also schon mehrere Monate früher ausgegeben worden sein. 
Nach ihrem Inhalt zusammengestellt ergeben sich folgende Hinweise Werlhofs (in Klammern steht das Datum der betreffenden Briefe):

1. Der mutmaßliche Autor des Werkes L'homme machine scheint Arzt und Schweizer zu sein (8. Dezember 1747), er ist - wegen seines Stiles wohl nicht in Frankreich geboren, kennt Hallers Gedichte und ist Arzt (15. Dezember 1747). Hallers Verdacht auf König «hat einen sehr hohen Grad von Wahrscheinlichkeit» (18. Dezember 1747) und «es ist zu befürchten, daß sich dieser Verdacht als wahr erweist» (22. Dezember 1747). La Mettrie allein ist nicht der Autor, ein Deutscher oder Schweizer muß beteiligt sein (17. Januar 1748). Alle späteren Briefe weisen nur noch auf La Mettrie als Autor hin. Ergebnis: Zur Zeit der ersten Rezension in den Göttingischen Zeitungen von gelehrten Sachen war über den Autor des Homme machine nichts Sicheres bekannt.

2. Nachrichten Werlhofs über diese früheste Besprechung: Auf Weisung von Münchhausens verfaßte Augspưrg einen Auszug aus dem Homme machine, den er sofort seinem Auftraggeber zustellte (8. Dezember 1747) ${ }^{13}$. Werlhof drängte, mit einer Anzeige oder Besprechung nicht zuzuwarten (29. Dezember 1747). Augspurgs Rezension wäre für den Zweck genügend (5.Januar 1748). Aufträge des Kurators von Münchhausen glaube Augspurg nicht zurückweisen zu dürfen, er führe sie aus, so gut er könne. La Mettrie war zu schlau für ihn. Haller brauche nicht alles ihm vom Kurator Übersandte zu drucken, sofern es nicht so ist, wie es nach seinem Empfinden hätte sein sollen (31. Mai 1748). Ergebnis: Die erste Besprechung des Werkes L'homme machine in den Göttingischen Zeitungen von gelehrten Sachen (1747, S.905-907) ist von Augspurg geschrieben, nicht von Haller, der sich - wahrscheinlich nach genauerer Kenntnis des Werkes - nicht ganz befriedigt davon zeigte.

\section{L'homme machine in Briefen von G.L.Schmid an J.G.Zimmermann und A.v. Haller}

Ein kurzer Briefwechsel zwischen A.v. Haller und J.St. Bernard, den ich 1966 publizieren konnte, hatte vorwiegend einige unveröffentlichte Konsultationen Boerhaaves zum Gegenstand. Darüber hinaus ergab sich

13 Auf die Weiterleitung des Auszuges an Haller kann ein Brief des Kurators von Münchhausen bezogen werden; in seinem eigenhändigen Schreiben vom 15. Dezember 1747 (Mss. Hist. Helv. XVIII. 62, Nr. 204) heißt es: «... Der anschluß [d.h. die Beilage] dependiret von Ew. Hochedelgeb. Gutbefinden, ob sie selbigen gefastermaßen [d.h. so 
aus dem damaligen Briefwechsel auch ein interessanter Hinweis auf die Entstehungsgeschichte der Broschüre L'homme machine von de La Mettrie. Bernard nannte in einem Brief vom 26. Februar 1752 als eigentlichen Urheber dieser Schrift und als Zuträger von Nachrichten über Haller einen angeblich aus Basel stammenden Schweizer namens Schmith von Auenstein. Haller wußte darüber schon Bescheid, denn er antwortete Bernard am 9. März 1752: «Ich kenne Amsteins oder Schmids Geschichte, dies ist nämlich dessen wahrer Name. Er lebt beim Fürsten in Gotha, ist auch Schweizer, aber nicht mein Mitbürger und weder mein Schüler noch mein Freund, noch sah ich ihn je.Aber ich will nicht machen, daß er durch mich erfährt, wie groß sein Anteil an dieser unanständigen Sache war.»

Auffälligerweise verschwieg Haller damals Bernard, daß er mit Schmid schon seit November 1749, also seit mehr als zwei Jahren, in ziemlich lebhaftem Briefwechsel stand und daß dieser ihm - vermutlich auf eine direkte Anfrage Hallers hin - bereits im Januar 1751 seine frühere Verbindung mit de La Mettrie zugegeben hatte. Aus der Matrikel der Universität Leiden konnte belegt werden, daß Schmid und de La Mettrie im Sommer 1747 Hörer der dortigen medizinischen Fakultät waren. Unklar blieb einzig, woher Schmid seine Informationen über Haller hätte haben können. Als einen möglichen Vermittler von Nachrichten über Haller wies ich auf Schmids Landsmann Johann Georg Zimmermans hin, der als Hallers Hausgenosse in Göttingen lebte. Ausdrücklich fügte ich jedoch hinzu, daß für eine solche Annahme keine Beweise bekannt wären.

Meine vorstehend zusammengefaßten früheren Ausführungen erfordern eine chronologische Ergänzung; Zimmermann wurde in Göttingen am 15. September 1747 immatrikuliert, als Tag seiner Ankunft in Göttingen nennt Hirzel (1882, p. CCXLIX, Anm.3) den 1.September 1747. Hätte ich dieses Datum schon früher beachtet, so würden allein schon zeitliche Gründe eher gegen als für die Annahme gesprochen haben, daß Zimmermann seinem Landsmann Schmid Informationen über Haller hätte zukommen lassen können. Zum mindesten wären sie nicht für das L'homme machine benannte Büchlein benutzbar gewesen, denn dieses erschien ja schon vor dem Ablauf des Jahres 1747.

Seither ist mir ergänzendes Material über die Verbindungen zwischen Schmid, Zimmermann und Haller bekannt geworden. In J. G.Zimmermanns

wie er abgefaßt ist] denen gelehrten Zeitungen einverleiben wollen.» Haller war danach also in seinen Dispositionen völlig frei. Im speziellen Fall stand er offensichtlich stark unter dem Einfluß Werlhofs. 
Nachlaß, den die Niedersächsische Landesbibliothek in Hannover verwahrt, stieß ich bei der Suche nach Haller-Dokumenten auch auf eine große Sammlung von Schreiben Schmids an Zimmermann. Die 480 Blätter umfassende Briefreihe aus den Jahren 1747-1785 ist im Katalog (S. 25, Nr. 83) charakterisiert als «von besonderem Interesse für die Geschichte bedeutender schweizerischer Persönlichkeiten jener Zeit». Im Hinblick auf Hallers Diskussionen mit de La Mettrie wählte ich aus diesen Briefen die der Jahre 1747-1752 aus. Es handelt sich dabei um insgesamt 50 Schreiben mit 167 Seiten Umfang; außer drei englischen sind alle in französischer Sprache abgefaßt. Ob auch die späteren Briefe Schmids an Zimmermann noch medizin-historisch Interessantes enthalten, konnte ich aus zeitlichen Gründen nicht prüfen. Die Wahrscheinlichkeit ist gering, da Schmid nach allem, was über ihn bekannt ist, nicht ärztlich tätig war. Daß Schmid auch mit Haller Briefe wechselte, wurde schon einleitend erwähnt.

Alle die Mitteilungen Schmids im Wortlaut zu veröffentlichen ist unnötig. Vollständig wiedergegeben sind nur sein erstes Schreiben an Zimmermann, weil es zu Schmids Charakteristik beiträgt, und ein späterer Brief vom 27.Juni 1749, der eine zentrale Bedeutung in der Diskussion um Schmids Anteil am Zustandekommen von de La Mettries L'homme machine hat. Im übrigen führe ich hier nur die Briefstellen an, die Beziehungen zwischen Schmid und de La Mettrie und dessen Streit mit Haller betreffen; einige wenige Sätze gelten Schmids Zukunftsplänen.

Wieder sind biographische Angaben über die Briefempfänger A.v. Haller (1708-1777) und J.G.Zimmermann (1728-1795) unnötig, beide werden in den medizin-historischen Hand- und Lehrbüchern genügend berücksichtigt. Dagegen müssen den Briefstellen einige Notizen über Georg Ludwig Scнмid (1720-1805) vorausgeschickt werden. Als Quellen dafür dienten mir die sehr knappe Lebensbeschreibung von Lutz (1812, S.475), einige Hinweise von Bäschlin (1917, S.84, 166, 169 ff.) und Schmids eigene Angaben über seine Familie und deren Herkunft.

Georg Ludwig Schmid wurde als Sohn eines Predigers am 12. März 1720 in Auenstein (Aargau) geboren. Von seinen Jugendjahren ist nichts Besonderes zu melden. Als Student wandte er sich nach Frankreich. Dort wurde er mit de La Mettrie bekannt. Er suchte in Beziehung zu wissenschaftlich berühmten Männern und zu adligen Familien zu kommen, weil er glaubte, auf die Weise eine angesehene Stellung zu finden. Anscheinend hat er sich damals außer für die Medizin besonders für Physik und deren Grundlagen interessiert. Ob er besonderen Anlaß hatte, 1747 zur weiteren Ausbildung 
nach Leiden zu ziehen, ist unbekannt. Sicher ist, daß er am 16. März 1747 dort als stud. med. immatrikuliert wurde; auch in Leiden traf er wieder mit de La Mettrie zusammen (Hintzsche, 1966). Lange kann Schmid, der mittlerweile im 28. Lebensjahre stand, nicht in Holland geblieben sein. Die nachstehend veröffentlichten Briefe an Zimmermann zeigen, daß er über Frankfurt und Heidelberg nach Straßburg reiste, angeblich hat er auch die Höfe in Mannheim, Zweibrücken und Zabern besucht; es kann sich dabei aber nur um sehr kurzfristige Aufenthalte handeln. Ohne seine Ausbildung abgeschlossen zu haben, war er damals auf dem Wege nach Haus, weil ihm sein Vater weitere Mittel für die Fortsetzung der Studien verweigerte. Von Straßburg aus gelang es ihm, eine Stelle im Sachsen-Weimarischen Hof dienst zu erhalten, die er zu Anfang des Jahres 1749 antrat. Von seiner damaligen Tätigkeit schrieb er später in einem Brief an Tscharner: «Ich beaufsichtigte die Erziehung des jungen Herzogs ${ }^{14}$ und war zugleich in verschiedenen Kommissionen. Herr Graf von Bünau ${ }^{15}$ würdigte mich seines Vertrauens und seiner Familiarität» (Bäschlin 1917, S.170). 1756 kehrte Schmid mit dem Titel eines Hofrates und einer Pension in die Heimat zurück, wo er sich literarisch in der Ökonomischen Bewegung betätigte. Er wurde im März 1759 zum Ehrenmitglied der Ökonomischen Gesellschaft in Bern ernannt und leitete von 1761 an als Präsident deren Zweiggesellschaft in Aarau. Seine weitere wissenschaftliche Tätigkeit sei hier übergangen, weil sie ihrem Inhalt nach zu weit vom Thema abliegt. Schmid unterhielt einen lebhaften Briefwechsel mit bedeutenden Männern im In- und Ausland; er starb am 30. April 1805 in Lenzburg.

Über die Abstammung seiner Familie schrieb G.L.Schmid am Schluß seines ersten an Zimmermann gerichteten Briefes vom 21. Dezember 1747: Unsere Familie ist trotz ihrer gegenwärtig eher bescheidenen Lage eine der ersten der Schweiz. Sie ist ein Zweig des Urner Geschlechts der Schmid von Bellikon und war früher im Besitz von Auenstein. Dazu wäre festzustellen, daß (nаch н в L s, 6. Band, S. 209, Nr. IV) die Schmid von Bellikon ursprünglich aus dem Eschentale stammten und sich 1566 in Uri einbürgerten; nachdem sie 1640 Schloß und Dörfchen Bellikon im Aargau erworben hatten, wurden sie 1646 von Kaiser Ferdinand III. in den Reichs-Ritterstand erhoben. Wäre G.L.Schmids Meinung über die Herkunft seiner Vorfahren richtig, so könnte die Abzweigung von der Urner Stammlinie kaum

14 des noch minderjährigen Ernst August II. Konstantin.

15 Graf Heinrich von Bünau (1697-1762) war zunächst Statthalter und von 1748-1758 Erster Minister des Herzogs Konstantin. 
vor der 2. Hälfte des 16. Jahrhunderts erfolgt sein. Die Herrschaft Auenstein war aber schon seit 1491 in den Händen der Berner Familie von Mülinen und diese veräußerte den Besitz 1644 an den Berner Hans Ludwig von Erlach (н в LS , 1. Band, S.473). Nach derselben Quelle (6. Band, S.201) ist der Name Schmid (und seine verschieden geschriebenen Varianten) im Aargau seit dem 14. Jahrhundert an zahlreichen Orten sehr stark vertreten; allein in der Stadt Aarau kennt man mehrere Stämme dieses Namens, die jedoch verschiedener Herkunft sind. Unter diesen wird als Nr.1 Georg Ludwig Schmid von Aarau mit den Lebensdaten 1720-1805 angeführt, also der Mann, mit dem wir uns hier zu befassen haben.

Schmid selbst gab noch eine andere Version über den Ursprung seiner Familie an, entweder weil ihm Bedenken gegen die eben erwähnte Ableitung gekommen waren oder weil er - für den Fall einer Stellenannahme in England - sich mit dieser Nation verbunden zeigen wollte. Im Brief vom 1.November 1749 an Zimmermann schrieb Schmid, er sei englischen Blutes und stamme von den Smith von Avensten oder Aventon in der Grafschaft Glocester ab; diese hätten bei Gelegenheit von Wirren ihr Vaterland verlassen und Wohnsitz in Aarau genommen. Aus dieser Ableitung seiner Herkunft erklärt sich wohl auch die vereinzelt vorkommende Briefunterschrift Smith d'Avenstein oder d'Avenston (letztere Form nur in einem englisch geschriebenen Brief).

Wenn jemand so ganz ohne Hemmungen zwei grundverschiedene Möglichkeiten seiner Abstammung nennt, ist ziemlich sicher, daß beide falsch sind, was dem Schreiber der hier benutzten Briefe nicht unbekannt gewesen sein dürfte. Es war eine bewußte Vorspiegelung falscher Tatsachen, um leichter in der Welt der oberen Zehntausend Fuß zu fassen. Darf man trotzdem den Inhalt seiner Briefe als Quelle für eine historische Studie gelten lassen? Ich behaupte ja! Vieles in den Briefen ist richtig, weil es einerseits psychologisch verständlich ist, andererseits auch aus Material fremder Herkunft kontrolliert werden kann. Unzuverlässig ist vor allem, was der Briefschreiber über sich selbst anführt, denn er versucht, von seiner Persönlichkeit ein Bild zu geben, das den Empfänger der Briefe zu seinen Gunsten beeinflussen soll. Solche Menschen machen meistens den Fehler, zu glauben, es wäre leicht, andere zu täuschen. Die dazu angewandten Mittel sind gewöhnlich recht harmlos: Man regt an, Adressen oder Briefteile von anderer Hand schreiben zu lassen, schiebt einen angeblichen Freund vor, den man sprechen läßt, wenn man sich selbst äußern will, und ähnliche Methoden, wie sie uns in der Briefen von G.L.Schmid begegnen. In jedem 
Fall ist es ein Leichtes zu bemerken, wo Unwahrheiten beginnen. Dabei bleibt dann aber doch genug Interessantes, das uns ermöglicht, den Briefschreiber zu durchschauen, sein schlechtes Gewissen zu fühlen und daraus die erforderlichen Konsequenzen in der Beurteilung ganzer Briefe oder Teile derselben zu ziehen. Auch solche Briefe können also zu einer verwertbaren Quelle historischer Forschung werden, wenn wir sie nur, speziell hinsichtlich der Person des Briefschreibers, richtig werten. Das sei an einem Beispiel dargelegt: Wie kam der stud. med. Georg Ludwig Schmid zu dem Namen eines Mr. d'Avenstein, mit dem die meisten seiner Briefe unterzeichnet sind? Dazu verfolgen wir einige Briefunterschriften samt den dazu gehörigen Daten:

$\begin{array}{ll}\text { 7. Februar 1748 } & \text { G. L. Schmid d'Auenstein } \\ \text { 25. Juni } 1748 & \text { G. L. S. d'Auenstein } \\ \text { 30. November 1748 } & \text { G. L. d'Avenstein } \\ \text { 19. März } 1749 & \text { d'Avenston (englischer Brief) } \\ \text { 27. Dezember 1749 } & \text { d'Avestein } \\ \text { 6. Juli } 1750 & \text { Smith d'Avenstein }\end{array}$

Mit der in der Schweiz üblichen Beifügung des Herkunftsortes (heute des Heimatortes) war der richtige Name G.L.Schmid von Auenstein; der Familienname stand dann noch kurze Zeit als S. unter den Vornamen, später verschwand er ganz, die Herkunftsbezeichnung ersetzte ihn, man war Herr G.L. von Auenstein, in französischer Schreibung M. d'Auenstein bzw. - auch dieses noch korrigiert - M. d'Avenstein oder d'Avestein. Alles keine Kunst, man muß nur etwas auszulassen wissen, dann wird aus einem schlichten Georg Ludwig Schmid, der aus Auenstein ist, ein Edelmann des Namens d'Avestein; nur die Gesinnung entspricht nicht gerade diesem Vornehmtun, das mit der ständigen Sorge, entdeckt zu werden, erkauft wird. So viel als Beitrag zur Charakteristik von G.L.Schmid.

Gewiß würde die Beurteilung mancher Stellen in den Briefen Schmids erleichtert werden, wenn auch die an ihn gerichteten Schreiben von Zimmermann bekannt wären. Es war aber nicht festzustellen, ob und - wenn ja wo sich solche erhalten haben. Die Wahrscheinlichkeit ist eher gering, denn Schmid schrieb in einem Brief vom 16. Februar 1751 an Zimmermann «Je brule toutes les lettres de conséquence.» Am 13. Januar 1752 erwähnte er dann allerdings einmal, er hätte einen Brief von de La Mettrie aufbewahrt, in dem dieser ihm schrieb, Schmid sei der Hauptanlaß für de La Mettries Berufung nach Berlin gewesen. 
Andererseits scheint es, daß Zimmermann seine Korrespondenz sorgfältig sammelte. Es fanden sich keine Hinweise dafür, daß etwa Briefe Schmids verloren sind oder vernichtet worden wären; selbst Schreiben, deren Verbrennung vom Absender ausdrücklich gewünscht wurde, sind erhalten geblieben.

Die nachstehend publizierten Briefe und Briefteile sind wörtlich wiedergegeben. Die Schreibweise blieb unverändert mit Ausnahme zweier Schriftzeichen. Schmid pflegte in französischen Worten ein am Schluß stehendes $\mathrm{i}$ als j zu schreiben, also moj statt moi usw. Der Lesbarkeit wegen ist an solchen Stellen stets i gesetzt. Eine weitere Besonderheit betrifft die Schreibung des y; vor allem zwischen Vokalen aber auch sonst gelegentlich steht statt des y ein $\mathrm{j}$ oder ein $\mathrm{i}$, beide dann mit Trema geschrieben. Im Druck steht dafür regelmäßig y.

Schmid an Zimmermann:

Heidelberg le 21. Dec.1747.

Monsieur,

Je viens de recevoir une letre de Mr. Stapfer ${ }^{16}$, dans laquelle il me marque ce que vous avez eu la bonté de lui mander à mon sujet. Votre souvenir, et la maniere avantageuse dont vous le faites me fait beaucoup d'honneur et de plaisir. Puisque vous voulez bien vous interesser pour moi, je vous ferai un plus grand detail de mes circonstances, et de mes vues, que je n'ai fait jamais.

Vous sentez bien, Monsieur, que j'eusse satisfait egalement mon ardeur pour les Sciences, et mon envie à conoitre les grands hommes, si j'eusse pu etre assez heureux pour pouvoir voir Mr. Haller à Göttingue. C'etoit mon premier plan, que de m'arreter à cette Université en revenant de la Hollande. Mais mon Pere commençant à me refuser des Secours m'obligea de partir pour Francfort pour y accepter un Emploi, qu'on m'avoit ofert. Depuis je n'ai pas osé m'éloigner plus de ma patrie, et faute de moyens je ne pourrai pas le faire à l'avenir sans quelque ocasion favorable, malgré le grand envie, que j'ai de le faire.

Vous savez une partie de ma vie, et je n'ai pas besoin de vous en dire quelque chose, que depuis le tems, que j'ai quité la Suisse. Je continuai en France avec beaucoup d'aplication l'étude de la Medecine et de la Physique, et j'employois les heures perdues à frequenter le monde, ayant eu beaucoup de bonnes conoissances. Les Jesuites, parmi lesquelles j'avois beaucoup d'amis, voulurent m'atirer dans leur Societé, et Mr. de Fontenelle voulut me faire Catolique pour m'atacher à l'Academie. Ayant fait ce que je pretendois faire à Paris, et ne voyant point de jour pour un etablissement je passois à Leyde ou je continuois mes etudes favorites. Mr. le Comte de Twikel, Mr. le Baron de Schwarzenberg et Mr. de Changuyon voulurent me porter, comme Mr. le Comte de Hohenlohe-Kirchberg avoit fait avant que j'avois quité la France, successivement à mener leurs fils dans leurs voyages. Mais c'etoit sous des conditions si onereuses d'un coté quoique avantageuses de l'autre, que j'ai eté

16 Johannes Stapfer (1719-1801) war wie Schmid und Zimmermann aus aargauischer Familie; er schloß seine theologischen Studien 1745 ab und war dann Hauslehrer und Reisebegleiter der Gebrüder Tscharner aus Bern. 
plus fidele aux Sciences, qu'à la Fortune. Pour me metre à l'abri de mon Pere je venois donc à Francfort pour y pratiquer sous Mr. Muller ${ }^{17}$, et pour prendre en meme tems la place qu'avoit Mr. de Lohenschiold relative à la Politique. Mais le libraire etoit si deraisonnable, que je le quitois en peu de tems pour aller à Heidelberg pratiquer sous Mr. Nebel ${ }^{18}$, et pour m'atacher à la Cour Palatine. C'etoit une idée que Mr. le Baron de Grevenbroek Ministre de l'Electeur en France m'avoit donnée. L'un et l'autre alloit à mon souhait. Je fis une entrée fort agréable à cette Cour. Les Ministres et les principaux Seigneurs etoient pour moi, et j'y avois surtout un excellent ami Mr. le Comte de Schall. Je frequentois regulierement la Cour, et je fus bien reçu de l'Electeur, qui avoit donné parole de me declarer Conseiller de son Administration et Professeur en Physique de l'Université de Heidelberg avec survivance d'une Chaire en Medecine remplie actuellement par le premier Medecin de l'Electrice. Les Jesuites, qui sont toutpuissans, et qui ont des vues particulieres, ont trouvé moyen de faire un Scrupule de Conscience à l'Electeur de placer un Protestant, et de faire manquer tout: Conoissant à present mieux la Cour et ce Pays je n'en suis pas trop faché. J'ai dessein de quiter Heidelberg et d'aller à Strasbourg. J'y verrai mes amis je tacherai de profiter de Mr. Sachs ${ }^{19}$, et je m'aprocherai de ma Patrie, si je suis assez malheureux pour n'avoir point d'emploi jusques printems prochain. Je vous avoue, que cette idée me fait de la peine. Ce n'est pas que je n'aime point ma Patrie. Mais vous la conoissez comme un Theatre fort borné, et peutetre savez vous aussi, que j'ai le malheur d'avoir un Pere, qui n'a point les memes Sentimens que moi.

Vous avez eu la bonté, Monsieur, de parler de moi à notre illustre compatriote Mr. Haller. A ce que vous marquez à Mr. Stapfer il sera meme porté à prendre mes interets. Je deplore réellement le malheur de n'etre pas connu de lui. La conformité de mes sentimens, de mes inclination etc. avec ceux de Mr. Haller n'auroit point manqué de me metre dans ses bonnes graces, et peutetre que j'aurois eté assez heureux de gagner l'estime d'un homme, que je ne regarde pas seulement comme l'ornement de notre Siecle, mais que je me suis proposé pour mon modele encore en particulier.

Je n'ai point d'autre moyen de me faire conoitre à Mr. Haller que par D'autres de mes amis. En voici quelques uns: Mrs. Bernoulli, Clairaut, de Reaumur, de Mairan, Calandrin, Jallabert, Cramer, Abauzit, le Monnier le Medecin, Castillon, de la Metrie, Herrenschwand, Muller, Muschenbroek les deux freres, Allamand, Nebel, Schœpflin, d'Olenschlaguer, de Gaufecourt, Tarin, le P. Patouillet, le Baron de Thoms, Rabours, S.E. le Comte de Loss, Euler, le Fevre, de Fontenelle, de Chapeaurouge, le Marquis d'Itre, de Hagedorn, l'Abbé Nollet, le Baron de Wachtendonk, l'Abbé de Gua etc. Si Mr. Haller est en commerce avec quelqu'un d'eux il peut s'informer de moi, ou je me ferai adresser à lui. Un de mes amis de Leide sera revenu dans votre Pays. C'est Mr. de Berger fils de Mr. de Berger à Zelle. L'on m'a trouvé jusqu'ici capable de remplir des emplois assez diferens, et il n'y a pas longtems, que Mr. d'Olenschlaguer a voulu me donner pour Secretaire d'Ambassade à un Ministre de l'Empereur. Mais je vous avoue, que je prefererai un parti digne d'un philo-

17 Falls es sich um einen Arzt handelt, käme in Frage Gottfried Wilhelm Müller (1709-1799), der seit 1735 in Frankfurt tätig war; er arbeitete damals an anatomischen Kupfertafeln.

18 Wilhelm Bernhard Nebel (1699-1748), seit 1728 Professor der Medizin in Heidelberg, auch Hofarzt.

19 Johann Jacob Sachs (1686-1762) hatte seit 1738 die Cathedra pathologiae in Straßburg inne und erfreute sich einer ausgedehnten ärztlichen Praxis. 
sophe, à tout ce qu'il y a de brillant. La Medecine surtout a des charmes pour moi, qui m'ont fait perdre le trop grand atachement que j'avois pour les Sciences purement speculatives. La conduite de mon Pere m'a pourtant empeché jusqu'ici de prendre le degré de Docteur: mais je pourrai le faire quand je le trouverai necessaire aussitot.

Une remarque, que je n'ai jamais daigné, et que je ne daignerai jamais de faire en Suisse: mais qui suivant les idées des Allemands me pourroit servir beaucoup, et qui m'a deja rendu de grands services: c'est, que notre famille malgré l'Etat d'humiliation, dans lequel elle s'est trouvée par raport aux biens de la fortune, est pourtant une des premieres de la Suisse, puisqu'elle est une branche de ces Schmid de Bellike du Pays d'Ury, et qu'elle a possedé autrefois Auenstein.

Je sai, que Mr. Haller a tant d'occupations, que je n'ai point voulu lui adresser ce detail. Vous pouvez en faire, Monsieur, tel usage, que vous trouverez à propos. Je vous suplie seulement, de me faire la grace, de me faire part des idées de Mr. Haller sur mon sort. Si votre letre peut venir ici jusqu'au milieu au plus tard du mois prochain, elle me trouvera encore ici. Si vous ne pouvez absolument point me donner reponse jusqu'à ce tems-là, je vous prie de metre votre letre dans une incluse sous cette adresse: à Monsieur de VallTravers, Gouverneur de Mr. le Comte de Loss, chez Mr. le Dr. Beckler près de l'Intendance à Strasbourg. J'ai l'honneur d'etre avec beaucoup d'estime

Monsieur

$$
\begin{gathered}
\text { votre très-humble, très- } \\
\text { obeïss. Serviteur } \\
\text { Schmid d'Auenstein } \\
\text { chez Mr. le Dr. Schmedes. }
\end{gathered}
$$

\section{Schmid an Zimmermann:}

Heidelberg le 7. Fevr. 1748

Je vous suplie, Monsieur, d'avoir la bonté de me faire toujours part des occupations, des desseins, et des decouvertes de Mr. Haller. Vous ne pourrez me faire un plus grand plaisir. A-t-il abandonné tout à fait la poësie, et qu'en pense-t-il à present? Que pense-t-il des vers non-rimés? J'ai perdu un peu de vue l'histoire literaire de l'Allemagne. N'avez vous point quelques nouvelles dans vos quartiers?

Schmid an Zimmermann:

Strasbourg le 10.Avril 1748

Ce que vous venez de me mander de Mr. de la Metrie m'etonne. Je l'avois conu assez bien: mais je l'ai perdu de vue depuis quelque tems. Ne saurez vous point sous quel titre il a une pension du Roi de Prusse, et s'il est actuellement à Berlin. Je ne connois l'homme Machine que par la Gazette de Göttingue. Est-il si dangereux comme on le fait y passer?

\section{Schmid an Zimmermann:}

Strasbourg le 27. May 1748

J'avois pris la liberté de vous demander aussi des éclaircissemens touchant Mr. de la Metrie. Quelle raison dois-je donner de votre silence? Ma letre du 10. Avril ou votre reponse peut etre perdue. Peutetre, que j'ai touché un mot de mon voyage à l'armée. Que de telles considerations ne vous empechent point de m'ecrire.

Schmid an Zimmermann:

Strasbourg le 25. Juin 1748

Il est vrai, que j'ai eté lié avec Mr. de la Metrie. J'ai estimé son imagination vive et sa politesse, qui ne pouvoit pas manquer à un homme, qui etoit lié avec beaucoup de Seigneurs 
du premier rang. J'avois connu de ses amis à Paris. Mais je serois bien faché d'avoir eté une ocasion innocente du chagrin, que peutetre Mr. Haller a eu de voir son nom à la tete de la production de Mr. de la Metrie. J'en demanderois pardon à Mr. Haller. Si tout le monde, ou je me suis fait gloire de faire conoitre l'honneur de la Suisse, en faisant conoitre ses excellens ouvrages, devroit lui dedier des livres il seroit bien importuné. J'ai poussé mon gout si loin, que je suis devenu imitateur sans presque le savoir. Une bagatelle de ma façon, que Mr. d'Olenschlag a fait passer à ses amis à Ratisbone a eu de l'aprobation, parce qu'on avoit trouvé, que l'autheur copioit Mr. Haller. Il me semble, que Mr. Haller fait trop peu de cas de ses Poësies. Auroit-il pu rendre un plus grand service au Monde, que de remplir les hommes de sentimens nobles et genereux, et rendre la vertu aimable?

J'ai parlé bienserieusement, quand je vous ai demandé, si le livre de Mr. de la Metrie etoit dangereux. L'ouvrage ne m'etoit pas parvenu, et je conoissois l'Autheur. Pour faire un Ouvrage dangereux, il ne sufit pas de metre quelques traits d'une imagination vive. Il faut beaucoup de conoissances, et une grande force de raisonnement.

L'academie de Berlin aura de la peine à se soutenir, si elle se charge de toutes sortes de sujets. J'avois aussi tourné mes vues de ce coté-la, et je voyois beaucoup d'aparence à réussir. Mais je ne sais point, si je dois continuer. Cet etablissement peutetre n'est pas trop solide: Peutetre qu'à la fin il ne sera plus honorable.

\section{Schmid an Zimmermann:}

Strasbourg le 30. Nov. 1748

Je vous prie de témoigner à Mr. Haller combien je serai toujours rempli de véneration pour lui, et combien je m'interesse pour sa gloire, malgré que [je] n'oserai plus l'admirer que comme Poëte. Ma situation presente reveillera peutetre ma veine endormie, et Mr. Haller aura alors de mes nouvelles.

Je ne suis plus lié avec Mr. de la Mettrie. Je crois, que je suis destiné de soufrir meme à l'ocasion des imprudences de mes amis. Mes liaisons avec des Savans sont à present peu de choses, et je suis lié avec un monde tout à fait diferent. Je ne sai point comme la chose s'est faite: Mais je tacherai de renouveller mes liaisons.

Schmid an Zimmermann:

Weimar le 24. Janv. 1749

... Je n'aurois pourtant point fait le Courtisan, et j'aurois été plus fidele aux Sciences, si les façons d'agir de mon Pere ne m'eussent point obligé de chercher un deboucher à tout prix.

Les raisons, que j'ai eues de me cacher, ont été certaines circonstances de ma vie, que j'aurois été charmé de laisser dans l'oubli. Comme notre famille depuis sa sortie de l'Angleterre n'a pas toujours gardé son lustre, je crains, que certaines circonstances pourroient me faire du tort dans ma carriere presente.

(Aus einem undatierten, als fol.15 bezeichneten Blatt ist zu ersehen, daß Schmid an der Kavalierskrankheit des 18. Jahrhunderts erkrankt war. Er ersucht Zimmermann um unauffällige Zusendung der zur Behandlung erforderlichen Medikamente. Seine Lebensweise am Hofe sollte bei deren Gebrauch so wenig wie möglich geändert werden müssen, damit kein Verdacht aufkomme.) 
Vous m'obligez beaucoup, Monsieur, en prenant de certaines précautions par raport à ma vie passée. Si je suis un jour assez heureux pour vous entretenir de bouche, j'aurai l'honneur de vous metre au fait de mes raisons, et de justifier certaines demarches, qui pourroient sembler déplacées à quelqu'un, qui n'est pas au fait de mes desseins et de la perspective, que je puis avoir raisonnablement. ... Vous me ferez aussi plaisir de me donner les nouvelles, que vous pourez avoir de Suisse, et si vous voudriez vous informer de la situation de Mr. de la Mettrie à Berlin.

(Der Bericht über den Verlauf der Erkrankung Schmids erfolgt von hier an auf besonderen Blättern und in der Form von Mitteilungen über das Ergehen eines Freundes.)

Schmid an Zimmermann:

Weimar le 17. Fevr. 1749

Je vous suplie d'assurer Mr. Haller de mes très-humbles respects, et de lui témoigner combien je suis sensible à la bonté qu'il veut avoir de s'interesser pour moi. Tout consideré, il me semble, que je ne me trouve point dans des circonstances aussi délicates, que vous pensez. Voici mes raisons. C'est une chose déterminée, que nous descendons d'une fort bonne famille angloise, qui s'est soutenue encore assez par des Ministres et des Médecins à Arau. Je me suis donné pour un descendant de cette famille, qui ayant été élevé en partie en Suisse avoit achevé ses études à Paris, à Leiden et à Strasbourg. Voilà qui est dans les regles. Je n'ai caché, que mes Parens, ma situation à Berne, et que j'ai voulu me faire Médecin. Mon Pere m'a forcé de dire que je n'avois point de Pere pour cacher notre brouillerie. Il a été si dur, que de me refuser un secours pour me mettre en état de prendre possession de mon emploi, et sans la Comtesse de Leiningen je n'aurois pas été en état de venir ici. ... C'est par cette raison, que je vous prie, de me donner partout, pour ce que je me suis donné ici, et de passer sous silence le reste.

(Das einem Brief vom 12. März 1749 beiliegende Blatt 24 ist ein typisches Beispiel für die Primitivität der Verschleierungsversuche Schmids. Der Beginn lautet: Instead of letting you know, that my friend is cured etc. gegen Ende dagegen heißt es: I know not what to drink. The beer becomes (sic!) me ill ... I dare not without giving suspicion, drink a Tisane. What's to do?)

Schmid an Zimmermann:

Weimar the 19. March 1749

I thank you for the accounts you give me of Mr. Haller. You will oblige me, if you will do the same still when you will do me the Friendship to write to me.

Schmid an Zimmermann:

Weimar le 7. May 1749

Vous voulez me faire entendre, à ce qu'il me semble, que je passe pour l'Auteur de l'homme machine. Je me souviens, que vous m'avez témoigné deja une fois quelque chose d'aprochant. Mais j'ai cru le monde bien informé depuis ce tems-là. Tous les écrits publics tombent au moins sur la Metrie. Il m'importe pourtant beaucoup de savoir tout ce qu'on 
en pense, pour pouvoir prevenir de certaines impressions. Vous me rendrez ainsi un service réel, si vous voudrez me mander tout ce que vous savez des discours tenus sur mon compte, et me dire quelles personnes, dans quels endroits, et sur quel ton, ont parlé de moi.

Je suis faché, qu'on sache dans ma patrie ou je suis. J'aurois été infiniment mieux en pouvant me cacher. Ayant eu besoin de secours, j'ai été obligé d'articuler à mon Pere mon établissement, en le supliant en meme tems de garder le secret. Cette démarche ne m'a rien valu du coté de l'interet; je serois bien faché si j'avois lieu de me plaindre en meme tems de la dureté, et de l'indiscretion de mes plus proches. Vous m'obligerez infiniment, si vous me mandez encore sur cet article tout ce, que vous en savez avec tout ce qu'on en dit, et toutes les circonstances. Tous ces avis me fourniront d'excellentes regles pour ma conduite ici. Ils me sont d'une grande nécessité, et je ne puis les avoir, que de vous.

\section{Schmid an Zimmermann:}

Weimar le 14. Mai 1749

J'ai pris la liberté de vous prier par ma derniere il y a 8 jours, de me donner quelques éclaircissemens sur l'homme machine, et ce que vous en aviez coulé dans votre letre. Depuis ce tems-là j'ai trouvé dans la Gazette de Goettingue de cette année p. 293 un passage, qui me surprend, et qui me donne des soupsons, comme si l'on vouloit parler de moi ${ }^{20}$. Faitez moi l'amitié de dire à ce sujet tout ce, que vous savez. Je ne suis point fait pour m'engager jamais dans une querelle literaire, et infiniment moins encore d'abuser d'une confidence qu'on m'auroit faite. Ce sera seulement un avis dont je pourrois faire usage pour mon particulier. Je voudrois savoir, qui fait les Gazettes, quel autheur on soupsonne dans le passage en question, d'ou l'on a tiré cette anecdote, ou quelles raisons on a de presumer ce qu'on y dit? Vous voyez bien, que c'est une pure amitié, que vous me faites, sans exposer ni vous ni personne. Je vous en donne ma parole d'honneur.

Je vous aurai pour cette amitié autant d'obligations, que pour les autres, que vous avez deja eu la bonté de me faire...

\section{Schmid an Zimmermann:}

Weimar le 21. May 1749

J'ai pris la liberté de vous faire quelques questions touchant le passage des Gazettes de votre ville, que vous avez bien voulu copier. J'ai ainsi deviné juste malgré moi. Je suis étonné, et fort curieux de savoir quelles raisons Mr. H. peut avoir de me soupsonner. Faitez moi la grace de l'entretenir sur cet article, et me mander ses raisons.

Je serois encore bien plus étonné, si je devrois croire, qu'un homme, qui pense si bien, et pour lequel j'ai tant de respect, voudroit me charger d'un livre, qu'il croit si mauvais, pendant qu'il n'en a, que des presomptions. Mr. de la Metrie se fait gloire d'en etre le Pere: qu'on lui laisse une gloire aussi mince et aussi dangereuse. Il me semble, que la fortune meme a voulu designer l'Auteur. Le livre procura à la Metrie une pension de 1000 Ecus pendant que j'étois assez embarassé de ma personne.J'ajouterai encore, que ma petite vanité est presque choquée de voir, que Mr. H. me croit capable de metre tant de folies,

20 Die Stelle lautet: «So gewiß wir übrigens belehrt werden, daß der D. Julian Offrai oder sonst sogenannte Mr. de la M. der Verfasser des H.M. seye, so sehr sind wir nunmehr versichert, daß der wahre Verfasser in der That ein Schweizer, obwohl kein Schüler des Hrn. H. Hallers ist, dessen Nahmen wir auch anzeigen könten, wann wir es nicht aus gewissen Ursachen unterliessen.» 
et de pauvretés dans une production, comme il s'en trouve dans L.H.M. [L'homme machine]. J'ose me flater d'avoir produit un ouvrage plus methodique et plus fort, si la bonne morale, et la prudence m'eussent permis d'écrire sur un sujet, sur lequel un bon physicien ne se peut presque point acorder avec le theologien.

Faitez-moi la grace de faire gouter ces reflexions à Mr. H.

\section{Schmid an Zimmermann:}

Weimar le 27. Juin 1749.

Monsieur,

La nouvelle de la maladie de Mr. Haller, que vous m'avez donnée, m'a afligé beaucoup. Je ne doute point, que la Providence, qui veille sans cesse au bien du genre humain, le conservera pour la propagation de la verité. Je vous prie de lui témoigner mes sentimens, et mes souhaits, que je fais sans cesse pour sa conservation.

C'est avec beaucoup de chagrin, que je vois, que Mr. Haller a eu assez mauvaise opinion de moi, pour pouvoir me soupsonner Autheur du malheureux H.M. [Homme machine]. Je crois, que je vous en ai deja témoigné mon deplaisir, et que je vous ai assuré en honnete homme, que je ne l'ai point composé. L'Autheur meme ne l'a écrit, qu'après mon départ de Leide, et dans le tems, que j'étois à la Cour Palatine à Manheim. S'il a abusé de certaines choses, qu'il avoit trouvé dans mes papiers, je n'en suis pas la cause.

C'est encore par Zele pour la gloire de Mr. Haller, que j'ai été cause innocente de la fameuse dédicace. J'ai voulu familiariser les beaux esprits de Paris avec le plus grand Poëte des Allemands ${ }^{21}$. On a abusé d'une partie de mes traductions. Mr. de Voltaire meme l'a fait. Faut-il donc croire, que tout le monde abusera de nos bonnes intentions? L'ancienne maxime dit plutot, qu'il faut, que chacun aye preventionem viri boni.

Je supli instament au reste Mr. Haller de laisser tomber cette afaire. Je veux etre oublié et caché par raport à Mr. de la M. On se vange le plus noblement par le silence.

Il m'est tombé ces jours passés un écrit en main, qui porte le titre de machine terrassée ${ }^{22}$. On y parle de la Metrie, comme d'un homme, qui s'étoit empoissoné. Est-ce une fiction, ou seroit-il mort? Quelles nouvelles en avez vous?

Je suis avec beaucoup d'estime,

Monsieur,

votre très-humble

et très-obeïss. Serviteur

A.

Schmid an Zimmermann:

Weimar le 20. Oct. 1749

P.S. Je vous ai deja prié une fois, Monsieur, de me donner des nouvelles de Mr. de la Mettrie. Une petite brochure, qui m'est tombé entre les mains, le dit mort. Il m'importeroit de savoir s'il est en vie, et si ses circonstances sont bonnes. J'ai encore quelque chose à finir avec lui. Je vous prie de tacher de me donner des nouvelles detaillées de sa situation. Je n'ose pas m'en informer ici sans m'exposer.

21 Übersetzungen Hallerscher Gedichte ins Französische wurden damals auch von anderer Seite versucht; als Beispiel sei verwiesen auf Essai d'une Traduction en Prose des Poesies de Mr. Haller (Bibl. Rais., Vol. 39, P. 2, p. 389, Oct.-Dec. 1747). Übersetzer ist der Berner V.B.TscharNer (Göttingische Zeitung von gelehrten Sachen 1749, S. 370).

${ }^{22}$ Die Epître à Mlle A.C.P. ou la Machine terrassée ist referiert in den Göttingischen Zeitungen von gelehrten Sachen 1749, S. 696. 
Je ne saurois vous dire combien d'obligations je vous ai pour les soins, que vous avez voulu prendre de metre Mr. Haller dans mes interets.

Les raisons, que vous donnez, mon cher Monsieur, du choix de l'Angleterre, sont trop flateuses pour moi. Mr. Haller ne goutera-t-il pas encore la suivante? Comme je suis de sang anglois, et que je descends des Smith d'Avenston ou Aventon de la province de Glocester, qui se sont établis à Arau à l'occasion des troubles de leur patrie, est-ce que cette consideration ne me pourroit pas valoir de certaines facilités en Angleterre pour quelque emploi, que les étrangers ne trouvent point à l'ordinaire?

P.S. Puisque vous croyez, que Mr. Haller ne prendra pas mal, si je lui adresse quelques lignes, je vous prie de lui rendre l'incluse, et de l'instruire de bouche du reste. Je n'ai point voulu entrer la premiere fois dans un trop grand detail.

\section{Schmid an Haller:}

Weimar le 1. Nov. 1749

... N'ayant point pu avoir le bonheur de vous joindre, Monsieur, à Goettingue, comme je m'étois proposé en revenant de Leyde, je me suis servi de l'entremise de Mr. Zimmermann pour faire votre connoissance.

Mais comme cet ami me marque à present, que vous voulez, Monsieur, étendre vos bontés jusqu'à epouser mes interets, je me crois authorisé de vous en témoigner directement ma reconoissance...

Mr. Zimmermann est assez instruit de mes vues, et de mes circonstances pour vous en instruire, Monsieur.

\section{Schmid an Zimmermann:}

Gotha le 9. Janv. 1750

Que dois-je penser du contretems arrivé à l'égard de Berlin? Mr. de H. m'en parle dans des termes, comme s'il étoit determiné à refuser cette vocation. Que j'aurois souhaité, qu'il l'eut accepté! Il me semble mes interets meme mis à part, que je devrois le souhaiter pour l'avantage des Sciences. Je suis sur, qu'elle y gagneroient en tout.

Schmid an Haller:

Gotha le 9. Janv. 1750

J'ai été bien édifié et consolé par les sages reflexions, que vous avez eu la bonté, Monsieur, de me faire. Il me semble en examinant le fond de mon cœur, que ce n'est pas justement l'ambition, qui guide mes pas.

Schmid an Zimmermann:

Gotha le 1. Mars 1750

... Mr. de Haller auroit-il changé de sentiment à mon ègard ? Faitez-moi la grace de m'en éclaircir.

Schmid an Zimmermann:

Gotha le 6. Juill. 1750

... N'avez vous point de nouvelles de la Mettrie? Doit-il etre bien à Berlin?

\section{Schmid an Zimmermann:}

Gotha le 10. Nov. 1750

On veut avoir ici la nouvelle de la mort de la Mettrie. Des Courtisans de Berlin m'avoient assuré, qu'il avoit été bien avec le Roi. Est-ce que Mr. de H. n'est plus lié avec Mr. de Maupertuis? Ne lui a-t-il plus parlé de moi? Je me suis toujours fait de la peine à me servir de la Mettrie pour faire cette connoissance. 
Vous ne paroissés gueres prevenue pour Berlin. Toutes les anecdotes que j'en sais, confirment votre sentiment. Il vaut mieux etre employé dans les afaires du Roi, que membre de la Societé litteraire, titre très-équivoque. Les relations, que j'ai touchant la Mettrie, ne sont rien moins que favorables à ce bel-esprit en titre d'ofice. Pourquoi ai-je eu la foiblesse de vivre bien avec tout le monde! Mr. de H. m'écrit encore d'une façon à me faire repentir d'avoir vecu avec des libertins. Mais on en trouve tant, sous sa main, qu'il est impossible de les éviter.

Schmid an Zimmermann:

Gotha le 3. Janv. 1751

... J'ai reçu ce matin une lettre de Mr. de H. qui me fait part de celle de Mr. Wetstein ${ }^{23}$. Celle-ci contient à peu près un refus en forme. Les reponses de Mr. Kœnig disent à peu près la meme chose.

Il me semble meme, que je puis croire sans prévention, que ces Mrs. n'ont point de moi l'idée, qu'on en a eue il y a deja trois ans ailleurs. Ils me regardent sur le pied de ces jeunes Suisses, qui vont par toute la terre pour chercher fortune, et dont on ne sait à l'ordinaire que faire. J'en ai connu trop, et de trop près, pour n'avoir pas la présomption de me metre hors de cette catégorie. Je sens, que je saurois faire encore autre chose, que ce qu'on fait faire à l'ordinaire à nos compatriotes.

\section{Schmid an Haller:}

Gotha le 13. Janv.1751

Je suis infiniment sensible à la grace, que vous me faites en continuant de m'instruire, Monsieur, par vos conseils. Vos reflexions sur ma liaison avec la Mettrie ne sont que trop bien fondées. Mais elle a été si légere, si courte cette liaison; elle est brisée depuis si longtems, qu'elle ne pourra jamais me faire du tort auprès des gens raisonnables. Chacun auroit pu etre trahi par un homme, qui a voulu absolument dépayser le public, et passer pour Suisse. Je ne sais pas meme ou il aura pu pecher l'anecdote de Mr. Steiguer: je ne puis gueres lui en avoir parlé: car je ne me souviens nullement d'avoir connu un Steiguer de Wittighofen.

\section{Schmid an Zimmermann:}

Gotha le 25. Janv. 1751

... Je n'ai jamais perdu de vue le service de Prusse: mais je n'ai pas encore pu trouver un canal, qui m'ait contenté.J'ai afecté par plusieurs raisons de ne me jamais servir de la Metrie: à présent on vient me dire, qu'il est mort. En savés vous quelque chose?

P.S. Il me paroit par les dernieres lettres de Mr. de H. qu'il est rafroidi pour moi. En saurés vous deviner une raison? Je vous prie de me parler à cœur ouvert.

Schmid an Zimmermann:

Gotha le 16. Fevr. 1751

Je suis charmé d'aprendre, que je me suis trompé dans mes soupsons à l'égard de Mr. de H. Est-ce qu'il n'avoit point soupsonné les dificultés qu'il rencontre?

Vous m'accusés de vouloir tuer la Mettrie. Nous avons eu ici deux fois cette nouvelle comme certaine. La charité voudroit, que je la souhaitasse vraie: je crains, qu'il ne soit encore bien malheureux, s'il vit longtems. J'ai d'ailleurs encore une raison à en vouloir m'instruire. Je ne suis pas encore bien determiné si je ne devrois point renouer avec un

${ }^{23}$ Verleger wissenschaftlicher Werke in Amsterdam. 
Leistungen seines bedeutenden Landsmannes in Paris bekanntmachen zu wollen und behauptete im gleichen Brief, de La Mettrie hätte solche Schriftstücke mißbraucht (27.Juni 1749). Er fand Zimmermanns Dissertationsthema [De irritabilitate] interessant, weil er selbst in Holland darüber gearbeitet und mit Albinus einige Male davon gesprochen hätte; de La Mettrie hätte etwas von dem ihm durch Schmid Berichteten verstümmelt in den Homme machine aufgenommen (16. Mai 1751).

Im ganzen ist die Verteidigung von Schmid nicht ungeschickt; er verrät seine genauere Kenntnis der Verhältnisse aber doch immer wieder durch zu bestimmte Angaben über Einzelheiten, so daß jeder Leser gewiß Bedenken gegen etliche Briefstellen haben wird und das berechtigtermaßen, zumal wenn man Schmids sonstige Betrügereien zur Kenntnis genommen hat.

Auf zwei kluge Äußerungen Schmids sei zum Schluß noch hingewiesen: Als er verdächtigt wurde, den Homme machine verfaßt zu haben, betonte er, Arzt und Theologe könnten in den darin behandelten Fragen fast nicht übereinstimmen (21. Mai 1749). Kurz danach riet er Haller dringend, den Streit ruhen zu lassen; man räche sich am besten durch Schweigen (27. Juni 1749).

\section{Schlußbetrachtung}

Die Ergebnisse der Briefe Werlhofs und Schmids aus den Jahren 1747 bis 1752 lassen sich zwanglos zu einem Gesamtbild vereinigen. Im Schrifttum wird immer wieder die Ansicht vertreten, der Verfasser des Homme machine sei in der Person von de La Mettrie sehr bald erkannt worden (DE Saussure, 1949, S.431; Vartanian, 1960, S.7). Haller, den es ja wohl am meisten anging, brauchte im Gegensatz dazu 11/2 Jahre, ehe er sich der allgemeinen Ansicht anschloß, und das trotzdem er durch Zimmermann sicherlich die ersten Äußerungen Schmids zu dieser Frage lange vorher kannte. Bemerkenswert richtig ist das Urteil Werlhofs, de La Mettrie allein könne das Werk nicht geschrieben haben, ein Deutscher oder ein Schweizer müsse daran beteiligt gewesen sein. Das eben war G.L.Schmid, vielleicht schon vom früheren Aufenthalt in Paris her, sicherlich aber während der gemeinsam in Leiden verbrachten Zeit im Sommer 1747. Schmids französische Übersetzung der Hallerschen «Ode an Herrn D.Gessner» ermöglichte es de La Mettrie, daraus entlehnte Stellen in seine Zueignungsschrift des Homme machine einzubauen. Schmid war aber auch der Vermittler von 
wissenschaftlichem Stoff; er machte de La Mettrie mit den Ansichten von Albinus über die Irritabilität bekannt. Haller schrieb später mit Bezug auf de La Mettrie und seine Kenntnis von der Irritabilität (1756, T.1, p.90): «... je sais par des voyes sûres, qu'il tenoit tout ce qu'il pouvoit savoir là dessus, d'un jeune Suisse, qui sans être médecin, \& sans m'avoir jamais connu, avoit lu mes ouvrages, \& vu les expériences de l'illustre Albinus; c'est là dessus que la Mettrie a fondé ce sisteme impie, que ses expériences mêmes servent à refuter.» Damit hat Haller aber die Briefstelle etwas verändert. In Schmids Brief ist Haller in diesem Zusammenhange nämlich nicht genannt, doch besaß ja de La Mettrie von seiner Übersetzertätigkeit her einige Kenntnis von Hallers Ansichten. Schmids Äußerungen waren aber wohl aktueller und vielleicht auch damals schon etwas breiter fundiert als die Hallers, wie man ja heute überhaupt die von de La Mettrie vertretenen Anschauungen über die Irritabilität wegen ihrer umfassenderen Formulierung höher wertet als die Hallers (Vartanian, 1960, p.82 und 88; Lester S. King, 1966, p.XLVI). Abweichend von Vartanian ist aber doch festzustellen, daß de La Mettrie wirklich Informationen durch einen jungen Schweizer erhielt ${ }^{24}$, dies jedoch nicht über Hallers Schriften, sondern über die Meinungen (nicht Experimente!) von Albinus.

Die Herkunft der Anekdote über den Landvogt Steiger in Wittikofen bleibt weiterhin unklar. Schmid schreibt in seinem Brief vom 13.Januar 1751 nur, er könne sich nicht erinnern, davon gesprochen zu haben, gekannt hätte er einen Mann dieses Namens nicht. Diese Bemerkung bedeutet jedoch nicht viel: Eine Anekdote könnte man ja auch erzählen, ohne die darin erwähnten Personen zu kennen.

Zimmermanns Darstellung des Streites zwischen de La Mettrie und Haller in dessen Lebensgeschichte (1755, S.226 ff.) ist als zeitgenössische Quelle in der neueren Literatur zu wenig beachtet worden. Ohne Nennung des Namens ist der Anteil von Schmid am Zustandekommen des Büchleins L'homme machine dort klar und richtig dargestellt, wie ausdrücklich festgestellt sei.

Von größerer Bedeutung ist die Frage: Wann nahm Haller erstmals öffentlich zur Schrift L'homme machine Stellung? Schon zu Hallers Lebzeiten und nach seinem Tode galt seit Römer und Usteri (1789, 1.Band,

${ }^{24}$ Auf Grund meiner Publikation aus dem Jahre 1966 hat das Toellner (1967, S.137, Fußnote 29) bereits festgehalten. Zu korrigieren wären die Vornamen Schmids, die ich bei der ersten Nennung falsch angab als Johann Georg - offensichtlich durch die Vornamen Zimmermanns beeinflußt - richtig muß es Georg Ludwig Schmid heißen. 
S.250) bis heute die erste Besprechung in den Göttingischen Zeitungen von gelehrten Sachen auf das Jahr 1747 (108. Stück, S.905-907, Dezember 1747) als von Haller stammend, so zuletzt noch bei Vartanian (1960, p.200 und 253), Guthke (1962 a, S.160, Anm.52) und Toellner (1967, S. 137, Anm. 25 und 28). Dabei ist aber schon mit dem letzten Heft des Jahrganges 1747 oder ganz kurz danach eine Liste der Druckfehler publiziert, an deren Ende es heißt: «P. 905. vor dem Göttingischen Artikel ist das hochnöthige Zeichen * ausgelassen. Dieses bezeigt nicht nur einen fremden Artikel, sondern einen solchen, von dem der Direktor [d.h. Haller] bekannt machen will, daß er ihn nicht verfertigt, wovon in einem demselben zugelegten Lobspruche hier die Ursache liegt.» Guтнке (1962 a, S.40) meint zwar, diese Notiz sei «höchst unglaubwürdig»; er begründet seine Ansicht (a.a.O., S.160, Anm. 52), daß es sich um eine von Haller verfaßte Rezension handle, mit dem Hinweis, sie wäre im Index librorum (judiciorum), p.334v, angeführt, hätte also Haller zum Verfasser ${ }^{25}$. Soll man wirklich glauben, Haller hätte geschrieben: «Der Ungenannte preist in der Zueignungsschrift den vorzüglichen Wert des Vergnügens aus den Wissenschaften mit gar lebhafter und angenehmer Feder »? ${ }^{26} \mathrm{Zu}$ meinem Bild von Hallers Persönlichkeit paßt diese milde Ausdrucksform nicht, er war dazu viel zu mißtrauisch! Es war der arglose Augspurg, der das Referat schrieb, wie die Briefe Werlhofs beweisen, nicht Haller!

Wie schwierig es ist, sich von einer immer wieder kolportierten Ansicht frei zu machen, beweist die Äußerung von DE SAussure (1949, p.443) über die erste Rezension des Homme machine. Auch er hielt Haller für den Verfasser, schrieb aber, Haller wähle eine Form, die ihn nicht als Autor erkennen lasse. «Haller speaks of himself as if he were not the author of the article.» Im Text folgt dann eine Stelle aus der Besprechung zum Beweis für diese Ansicht; die Möglichkeit, daß Haller vielleicht gar nicht der Autor wäre, ist überhaupt nicht erwogen. In dem Gewirr der Pamphlete um den Homme machine galt es offenbar bisher als undenkbar, daß in einem Satz einmal wirklich das gemeint sein könne, was darin ausgedrückt ist.

Wenn wir also die erste Besprechung des Homme machine in den Göttingischen Zeitungen von gelehrten Sachen nicht als Meinungsäußerung Hallers ansehen dürfen, ist es vielleicht die zweite gewesen, die in Form eines in

${ }^{25}$ Im Gegensatz dazu hat der die Judicia aus dem Jahre 1747 enthaltende Band Haller Mss.40 in seinem Register keinen Hinweis auf den Homme machine.

${ }^{26}$ Vom Verfasser dieser Studie hervorgehoben. 
zwei Teilen gedruckten Briefes auf den Seiten 409-412 und 425-428 des Jahrgangs 1748 der gleichen Zeitschrift steht? Auch diese wird ja in Hallers Index librorum (judiciorum), p. 334 v, angeführt. Man weiß aber schon sehr lange, daß sie nicht von Haller stammt; er selbst gab davon Nachricht an die Leser der Zeitschrift (1753, 32.Stück S.303-304, im Berner Exemplar mit handschriftlichem $\mathrm{H}$ am Rande!). Von der zweiten Besprechung war nämlich eine französische Übersetzung erschienen, die den Unmut von de La Mettrie erregte und ihn zu einer Gegenschrift veranlaßte, die er allerdings an die falsche Adresse richtete. Ihr Titel heißt Epitre à mon esprit ${ }^{27}$. Haller sagte darüber in der schon früher erwähnten Besprechung des Eloge de La Mettrie, diese Epistel «ist die Frucht eines lächerlichen Zorns über zwey Stücke der Göttingischen gel. Zeitung, die einen hiesigen berühmten Philosophen zum Verfasser haben. ... Sie gehn den Hrn.v. Haller im geringsten nichts an.» Verfasser der zweiten Besprechung des Homme machine ist nach Zimmermanns Angabe (1755, S.229), wie auch Hirzel (1882, p. CCLVIII, Fußnote 3) und neuerdings Vartanian (1960, p.101) richtig schrieben, der Göttinger Professor der Philosophie Samuel Christian Hollmann, also wieder nicht Haller.

Tatsächlich liegt Hallers erste öffentliche Reaktion gegen das Buch L'homme machine erst in seinem an Reaumur gerichteten Brief vom 12. März 1749 vor, der dann sofort im Maiheft des Journal des savants gedruckt wurde. In diesem ist aber de La Mettrie nicht genannt, weil Haller damals noch immer Schmid im Verdacht hatte, Verfasser des Homme machine zu sein, wie aus den hier publizierten Briefen hervorgeht ${ }^{28}$.

Als ein Ergebnis von allgemeiner Bedeutung für die Haller-Forschung ist festzuhalten, daß Eintragungen im Index librorum (judiciorum) nicht sicher auf Hallersche Rezensionen hinweisen, wie an zwei Beispielen zu zeigen war. Es mag sein, daß ich es mit einem Spezialfall zu tun hatte. Beide Male stammt die Aufzeichnung der Band- und Seitenzahl der Rezensionen des

${ }^{27}$ Eine eigentliche Rezension dieses Werkes enthalten die Göttingischen Zeitungen von gelehrten Sachen nicht. Der im Jahrgang 1749, S.293, stehende Passus vom 17.April (nicht 17. Mai wie de Saussure 1949, 9., 441, schreibt) ist nur eine Ankündigung, aber keine Besprechung.

${ }^{28}$ Der Brief Hallers an Reaumur ist wieder abgedruckt im Journal Helvetique, Mai 1750, S.456-460. In der Einleitung heißt es dort: «Mr. de la Métrie,Auteur prétendu de l'homme machine... », wobei aber das um ein Jahr später liegende Publikationsdatum zu beachten ist. Abermals gedruckt findet sich Hallers Brief bei WoLf (1859, S.133/134, Anm. 74). 
Homme machine im Index judiciorum von der Hand J.G.Zimmermanns, der ja dieses Register anlegte; es wurde erst von $1750 \mathrm{ab}$ durch Haller selbst weitergeführt. Zimmermann wußte von Hallers Interesse an dem speziellen Thema, er kannte auch viele Einzelheiten darüber durch seinen Briefwechsel mit Schmid, deshalb könnte er diese Hinweise auf die Schrift L'homme machine aufgenommen haben. Es läge dann also einer der Fälle vor, von denen Guthke (1962 a, S.43) schrieb, es könne sich der Verdacht einstellen, «daß der Index nicht ein Register der Hallerschen Rezensionen allein, sondern auch andrer in den Göttingischen Zeitungen von gelehrten Sachen erschienener Besprechungen ist» ein nützlicher Verdacht, den er aber für falsch hielt - dieses Mal war er aber, vielleicht ausnahmsweise, berechtigt: Es gibt Eintragungen im Index judiciorum, die sich auf Rezensionen beziehen, die nachgewiesenermaßen nicht von Haller stammen. Man sieht nur, wie notwendig die Erschließung neuer Quellen auch für Fragen der Problemgeschichte ist, sie allein können uns von dem immer schwierigen Abwägen von Wahrscheinlichkeiten befreien und in vielen Fällen zu gesichertem Wissen führen.

\section{Quellen und Schrifttum}

A. Handschriftliche Quellen

I. Burgerbibliothek Bern

Haller-Korrespondenz, Mss. Hist. Helv. XVIII:

Briefe von G.L.Schmid, Band 45-47

Briefe von P.G. Werlhof, Band 59-61

Briefe des Freiherrn von Münchhausen, Band 62

Hallers Judicia librorum 1747, Mss. Haller 40

Hallers Index judiciorum, Mss. Haller 55

II. Niedersächsische Landesbibliothek Hannover

J. G.Zimmermanns handschriftlicher Nachlaß

Abt. A, Teil II, Nr. 83 Briefe von G.L.Schmid

B. Druckwerke

Adelung Johann Christoph, Fortsetzung und Ergänzungen zu Christian Gottlieb Jöchers allgemeinem Gelehrten-Lexico, 1. Band, Leipzig 1784.

BäschlıN G., Die Blütezeit der Ökonomischen Gesellschaft in Bern, I.Teil, Laupen 1917.

Göttingische Zeitungen von gelehrten Sachen 1747-1753.

Guthke Karl S., Haller, La Mettrie und die anonyme Schrift L'homme plus que machine, Etudes germaniques 1962, S.137-143. 
Guthke Karl S., Haller und die Literatur, Göttingen 1962 (a),188 Seiten.

Haller Albert De, Memoires sur la nature sensible et irritable des parties du corps animal, T.I, Lausanne 1756, 400 Seiten.

Haller Albert von, Bibliotheca anatomica, T.II, Tiguri 1777.

Hintzsche Erich, A.v. Hallers Korrespondenz mit Johann Stephan Bernard, Clio Medica 1 (1966) 324-340.

Hirsch August, Biographisches Lexikon hervorragender Ärzte, 6 Bände, Wien und Leipzig 1884-1888.

Hinzel Ludwig, Albrecht von Hallers Gedichte, Frauenfeld 1882, DXXXVI + 423 Seiten.

King Lester S., Introduction to A. Haller, First Lines of Physiology, New York/London 1966, S. IX-LXXII.

LutZ Markus, Nekrologe denkwürdiger Schweizer aus dem 18. Jahrhundert, Aarau 1812.

Panckoucke C.L.F. (ed.), Dictionaire des sciences médicales, Biographie médicale,T.6e, Paris 1824.

Römer J. J. und Usteri P., Des Herrn von Hallers Tagebuch der medicinischen Litteratur der Jahre 1745-1774, 3 Bände, Bern 1789-1791.

Saussure Raymond de, Haller und La Mettrie, J. Hist. Med.1949, S. 431-449.

Toellner Richard, Anima et Irritabilitas, Sudhoffs Arch. 51 (1967) 130-144.

Türler HeINrich et al. (ed.), Historisch-Biographisches Lexikon der Schweiz, 7 Bände, Neuenburg 1921-1934 (= HBLS).

Vartanian Aram, La Mettrie's L'homme machine, Princeton (N.J.) 1960, 264 Seiten.

Wolf Rudolf, Biographien zur Kulturgeschichte der Schweiz, 2. Cyclus, Zürich 1859.

Zimmermann Johann Georg, Das Leben des Herrn von Haller, Zürich 1755. 